TRANSACTIONS OF THE

AMERICAN MATHEMATICAL SOCIETY

Volume 288, Number 2, April 1985

\title{
VARIETIES OF AUTOMORPHISM GROUPS OF ORDERS
}

BY

\author{
W. CHARLES HOLLAND ${ }^{1}$
}

\begin{abstract}
The group $A(\Omega)$ of automorphisms of a totally ordered set $\Omega$ must generate either the variety of all groups or the solvable variety of class $n$. In the former case, $A(\Omega)$ contains a free group of rank $2^{\aleph_{0}}$; in the latter case, $A(\Omega)$ contains a free solvable group of class $n-1$ and rank $2^{\aleph_{0}}$.
\end{abstract}

1. Introduction. This work was prompted by the following question of A. Ehrenfeucht (see the footnote on p. 47 of [8]); I thank J. Mycielski for calling it to my attention:

Must the group of automorphisms of an ordered set have a free subgroup of rank $2^{\aleph_{0}}$ whenever it has a free subgroup of rank 2 ?

The answer is yes. In fact, if the group $A(\Omega)$ of automorphisms of the (totally) ordered set $\Omega$ fails to satisfy every nontrivial equational group law, then $A(\Omega)$ must contain a free subgroup of rank $2^{\aleph_{0}}$ (Theorem 5.6).

Phrased in the last way, the question suggests an analogous question for groups $A(\Omega)$ which do satisfy some equational laws. In the first place, one should ask what varieties (equationally defined classes) can be generated by the groups $A(\Omega)$. We will show that these are just the varieties $\mathfrak{S}_{n}$ of $n$-solvable groups, together with the variety $\mathfrak{G}$ of all groups (Theorem 2.10). Then we show that if $A(\Omega)$ generates $\mathfrak{S}_{n}, A(\Omega)$ contains a free- $\mathfrak{S}_{n-1}$ subgroup of rank $2^{\aleph_{0}}$ (Theorem 5.3). As a byproduct of this investigation, we can describe precisely the structure of a group $A(\Omega)$ if $A(\Omega)$ generates $\mathfrak{S}_{n}$ (Corollaries 4.3 and 2.6), thus generalizing a theorem of Chang and Ehrenfeucht $[\mathbf{1}]$ from the case $\mathfrak{S}_{1}=$ abelian. In the special cases that $A(\Omega)$ is transitive on $\Omega$, we characterize not only the group $A(\Omega)$ but the set $\Omega$ if $A(\Omega)$ generates $\mathfrak{S}_{n}$ (Theorem 3.2 and Corollary 2.6), thus generalizing a theorem of Ohkuma [10], again from the case $\mathfrak{S}_{1}$.

In many ways the most natural context for the study of $A(\Omega)$ is as a lattice ordered group. The lattice operations are defined pointwise: for $f, g \in A(\Omega)$ and $\alpha \in \Omega, \alpha(f \vee g)=(\alpha f) \vee(\alpha g)$, and dually. The questions posed above have analogues in the language of lattice ordered groups (l-groups), where the operations $\wedge, \vee$ together with the group operations can be used in equational laws for $A(\Omega)$. In Corollary 2.6 we show that the group $A(\Omega)$ generates $\mathfrak{S}_{n}$ iff the l-group $A(\Omega)$ generates $A^{n}$ (the $l$-group analogue of $\mathfrak{S}_{n}$ ), and in this case $A(\Omega)$ contains a free$A^{n-1} l$-subgroup of rank $2^{\aleph_{0}}$ (Theorem 5.2). Finally, the group $A(\Omega)$ generates $\mathfrak{G}$ iff the l-group $A(\Omega)$ generates either $\mathcal{N}$ or $\mathcal{L}$, where $\mathcal{N}$ is the join of all $A^{n}$

Received by the editors June 11,1984

1980 Mathematics Subject Classification. Primary 06A05; Secondary 20 B27.

${ }^{1}$ Work done while the author was on sabbatical leave at Simon Fraser University. 
and $\mathcal{L}$ is the variety of all $l$-groups (Corollary 2.8 ). In each case $A(\Omega)$ contains an appropriate free $l$-subgroup of rank $2^{\aleph_{0}}$ (Theorems 5.4 and 5.5).

2. The varieties of $A(\Omega)$. For general background in the theory of ordered permutation groups and $l$-groups, the reader is referred to Glass [2]. We deal first with varieties of $l$-groups. Let $A$ denote the variety of abelian $l$-groups, $A^{0}$ the variety of one-element $l$-groups, and, for each positive integer $n, A^{n}$ the variety of those $l$-groups $G$ which have an $l$-ideal $H$ (convex normal sublattice subgroup) such that $H \in A^{n-1}$ and $G / H \in A$ (see Martinez [6]). Let $\mathcal{N}$ be the join of all $A^{n}$. Then $\mathcal{N}$ is covered by the variety $\mathcal{L}$ of all $l$-groups $[\mathbf{4}, \mathbf{5}]$. We show that the variety of $l$-groups generated by $A(\Omega), l$-var $A(\Omega)$, is either $\mathcal{L}, \mathcal{N}$, or $A^{n}$ for some $n$.

We make use of the (orbital) wreath product, defined as follows. Let $(K, \Lambda)$ be an ordered permutation group, that is, $K$ is a subgroup of $A(\Lambda)$. For each $\lambda \in \Lambda$ let $H_{\lambda}$ be a group with $H_{\lambda}=H_{\mu}$ when $\lambda$ and $\mu$ lie in the same $K$-orbit. Let $H=\prod_{\lambda \in \Lambda} H_{\lambda}$. The wreath product $\left\{H_{\lambda}\right\} \operatorname{Wr}(K, \Lambda)$ is the splitting extension of $H$ by $K$ where conjugation by an element of $K$ permutes the indices of $H$ in the obvious way. If $K$ is a sublattice of $A(\Lambda)$ and each $H_{\lambda}$ is an $l$-group, then $G$ becomes an $l$-group with the order $e \leq h k$ (for $e$ the identity element, $h \in H, k \in K$ ) iff for each $\lambda \in \Lambda, \lambda<\lambda k$ or both $\lambda=\lambda k$ and $h_{\lambda} \geq e$. If each $H_{\lambda}$ is given as an l-subgroup of $A\left(\theta_{\lambda}\right)$ for some totally ordered set $\theta_{\lambda}$, the wreath product can be described another way. Let $\Omega=\left\{(\sigma, \lambda) \in\left(\bigcup_{\lambda \in \Lambda} \theta_{\lambda}\right) \times \Lambda: \sigma \in \theta_{\lambda}\right\}$, and order $\Omega$ lexicographically from the right. Then $\left\{H_{\lambda}\right\} \operatorname{Wr}(K, \Lambda)$ consists of all $g \in A(\Omega)$ having the form $(\sigma, \lambda) g=\left(\sigma g_{\lambda}, \lambda \bar{g}\right)$, where $\bar{g} \in K$ and $g_{\lambda} \in H_{\lambda}$ for each $\lambda$.

Of special interest are the iterated wreath products of the $l$-group $Z$ of integers (permuting itself), $\mathrm{Wr}^{0} Z=\{e\}, \mathrm{Wr}^{1} Z=Z, \mathrm{Wr}^{2} Z=\{Z\} \mathrm{Wr}(Z, Z)$, and, generally, $\mathrm{Wr}^{n} Z=\left\{\mathrm{Wr}^{n-1} Z\right\} \mathrm{Wr}(Z, Z)$.

An orbital of an ordered permutation group $A(\Omega)$ is the convexification of an orbit $\alpha A(\Omega), \alpha \in \Omega$. Note that if $\Omega_{i}$ is an orbital of $A(\Omega)$, then the restriction of $A(\Omega)$ to $\Omega_{i}$ is $A\left(\Omega_{i}\right)$, and $A(\Omega)$ is the full direct product of its restrictions to orbitals. A natural congruence on an orbital $\Omega_{i}$ is an equivalence relation on $\Omega_{i}$ whose classes are convex, which is respected by the action of $A\left(\Omega_{i}\right)$, and which satisfies certain technical conditions (see McClearly [7] or Glass [2] for details). For our purpose here, the important properties are that the natural congruences on any orbital form a complete tower under containment, and if $C_{i} \subseteq C^{i}$ are natural congruences such that $C^{i}$ covers $C_{i}$ (a covering pair) and if $C$ is a $C^{i}$ class, then $A(\Omega)$ induces a primitive component corresponding to $C$ and $C_{i}$ by restriction to $C$ followed by the natural homomorphism arising from the congruence $C_{i}$. The primitive component $K=K\left(C, C_{i}\right)$ is again an ordered permutation group, and either (i) $K$ is regular, that is, $K$ is a subgroup of the real numbers $R$, and the set permuted by $K$ is a union of cosets of $K$ in $R$, or (ii) $K$ is 0-2-transitive on one of its orbits, which means that if $\alpha<\beta$ and $\gamma<\delta$ are points of that orbit, then there exists $k \in K$ such that $\alpha k=\gamma$ and $\beta k=\delta$ (see McCleary [7] or Glass [2, especially Corollary 4.4.1]). It is also useful to note that if $C$ is a congruence, then $A\left(\Omega_{i}\right) \approx\left\{A\left(C_{\lambda}\right)\right\} \mathrm{Wr}\left(G, \Omega_{i} / \mathcal{C}\right)$, where $\left\{C_{\lambda}: \lambda \in \Omega_{i} / C\right\}$ are the $C$ classes and the induced group $G \approx A\left(\Omega_{i} / C\right)$ in case $A\left(\Omega_{i}\right)$ is transitive (though not in general).

We call an element $e<f \in A(\Omega)$ join irreducible if $f=a \vee b$ and $a \wedge b=e$ imply $a=e$ or $b=e$. 
LEMMA 2.1. If on one orbital of $A(\Omega)$ there are covering pairs $\left(C_{i}, C^{i}\right), i=$ $1,2, \ldots, n$, of natural congruences with $C^{i} \subseteq C_{i+1}$, and there are $C^{i}$ classes $C^{i}$ with $C^{i} \subseteq C^{i+1}$, and induced primitive components $K_{i}=K_{i}\left(C^{i}, C_{i}\right)$ all nontrivial, then $A(\Omega)$ contains an l-subgroup isomorphic to $\mathrm{Wr}^{n} Z$; moreover, we can assume the generator of the upper copy of $Z$ is join irreducible.

ProOF. We prove by induction that $A\left(C^{i}\right)$ contains a copy of $\mathrm{Wr}^{i} Z$. Since $K_{1}$ is nontrivial, there exists a join irreducible $e<g \in A\left(C^{1}\right)$. Then $g$ generates an infinite cyclic totally ordered subgroup, so $A\left(C^{1}\right)$ contains an $l$-subgroup isomorphic to $Z=\mathrm{Wr}^{1} Z$. Assume $A\left(C^{i}\right)$ contains an $l$-subgroup $H_{0}$ isomorphic to $\mathrm{Wr}^{i} Z$. We may identify $A\left(C^{i}\right)$ with a subgroup of $A(\Omega)$ fixing each point not in $C^{i}$. Let $C$ be the $C_{i+1}$ class containing $C^{i}$. Since $K_{i+1}$ is not trivial, there exists a join irreducible $e<g \in A\left(C^{i+1}\right)$ such that $C g \neq C$ (this follows from the technical definition of natural congruence; see $\left[7\right.$ or 2]). Then the set $\left\{C g^{j}\right\}$ is pairwise disjoint. Let $H_{j}=g^{-j} H_{0} g^{j}$. Clearly $A\left(C^{i+1}\right)$ contains the full direct product $\prod H_{j}$, and conjugation by $g$ permutes the indices of this product. Thus $A\left(C^{i+1}\right)$ contains an $l$-subgroup isomorphic to $H_{0} \mathrm{Wr} Z \approx \mathrm{Wr}^{i+1} Z$.

If $f \in A(\Omega), \operatorname{supp} f=\{\alpha \in \Omega: \alpha f \neq \alpha\}$ and, for any subgroup $G \subseteq A(\Omega)$, $\operatorname{supp} G=\{\alpha \in \Omega: \exists f \in G, \alpha f \neq \alpha\}$.

LEMMA 2.2. If some primitive component of $A(\Omega)$ is not regular then $A(\Omega)$ has l-subgroups $G_{n} \approx \mathrm{Wr}^{n} Z, n=1,2, \ldots$, such that if $n \neq m$, $\operatorname{supp} G_{n} \cap \operatorname{supp} G_{m}=\emptyset$.

PROOF. If $K$ is a nonregular primitive component, $K$ is 0-2-transitive on one of its orbits. Let $\alpha<\beta$ be any two points of this orbit. We construct $G_{n} \approx \mathrm{Wr}^{n} Z$ with $\operatorname{supp} G_{n} \subseteq(\alpha, \beta)$. There exists $k \in K$ such that $e<k$ and $\operatorname{supp} k \subseteq(\alpha, \beta)$. Hence $K$ contains $\langle k\rangle \approx Z$. If $\gamma k \neq \gamma$, the intervals $\left(\gamma k^{i}, \gamma k^{i+1}\right)$ are pairwise disjoint, and $(\gamma, \gamma k)$ contains supp $k^{\prime}$ for some $e<k^{\prime} \in K$. Then $\operatorname{supp} k^{-i} k^{\prime} k^{i} \subseteq\left(\gamma k^{i}, \gamma k^{i+1}\right)$ and $K$ contains the full direct product of the groups $k^{-i}\left\langle k^{\prime}\right\rangle k^{i}$. Hence $K$ contains an $l$-subgroup isomorphic to $Z \mathrm{Wr} Z=\mathrm{Wr}^{2} Z$. The construction is completed by induction. We can then choose disjoint intervals $\left(\alpha_{1}, \beta_{1}\right),\left(\alpha_{2}, \beta_{2}\right), \ldots$ and find $G_{n} \approx \mathrm{Wr}{ }^{n} Z$ with $\operatorname{supp} G_{n} \subseteq\left(\alpha_{n}, \beta_{n}\right)$.

We let $l$-var $G$ denote the variety of $l$-groups generated by $G$, and $g$-var $G$ the variety of groups.

LEMMA 2.3. $l$-var $\mathrm{Wr}^{n} Z=\mathcal{A}^{n}\left[\right.$ 4, Theorem 4.6] and $g$-var $\mathrm{Wr}^{n} Z=\mathfrak{S}_{n}$ (see Neumann [9, 22.24 plus 17.6]).

LEMMA 2.4. If $A(\Omega)$ has just one orbital and there are only a finite number of natural congruences, then $A(\Omega)$ can be embedded (as an l-group) in the iterated wreath product of its primitive components (see Glass [2]).

We also note the following connection between wreath products and varieties. If $H \in \mathcal{A}$ and each $G_{\lambda} \in \mathcal{A}^{i}$ then $\left\{G_{\lambda}\right\} \operatorname{Wr}(H, \Lambda) \in A^{i+1}$.

THEOREM 2.5. If $A(\Omega)$ has just one orbital, the following are equivalent:

(i) There exist at least $n$ nontrivial primitive components or some primitive component is not regular.

(ii) $\mathrm{Wr}^{n} Z \subseteq A(\Omega)$.

(iii) $A^{n} \subseteq l$-var $A(\Omega)$.

(iv) $\mathfrak{S}_{n} \subseteq g$-var $A(\Omega)$. 
PROOF. That (i) implies (ii) follows from Lemmas 2.1 and 2.2, while (ii) implies both (iii) and (iv) by Lemma 2.3. If there are no more than $m<n$ covering pairs of natural congruences and all primitive components are regular, then Lemma 2.4 implies $A(\Omega)$ can be embedded in the iterated wreath product of $m$ regular, hence abelian, l-groups, and so $A(\Omega) \in \mathfrak{A}^{m} \subseteq \mathfrak{S}_{m}$, contradicting both (iii) and (iv). Hence (iii) implies (i), and (iv) implies (i).

COROLLARY 2.6. If $A(\Omega)$ has just one orbital then the following are equivalent:

(i) There exist exactly $n$ nontrivial primitive components and all are regular.

(ii) $\mathrm{Wr}^{n} Z \subseteq A(\Omega)$ but $\mathrm{Wr}^{n+1} Z \nsubseteq A(\Omega)$.

(iii) $l$-var $A(\Omega)=A^{n}$.

(iv) $g$-var $A(\Omega)=\mathfrak{S}_{n}$.

COROLlary 2.7. For any $A(\Omega), l-\operatorname{var} A(\Omega)=\mathscr{A}^{n}$ iff $g$-var $A(\Omega)=\mathfrak{S}_{n}$.

COROLlaRY 2.8. For any $A(\Omega), l$-var $A(\Omega)=\mathcal{N}$ or $\mathcal{L}$ iff $g$-var $A(\Omega)=\mathfrak{G}$.

Proof. $\mathcal{N}$ is the join of all $A^{n}$ and $\mathcal{L}$ covers $\mathcal{N}$. Hence $l$-var $A(\Omega)=\mathcal{N}$ or $\mathcal{L}$ is equivalent to the statement: for all $n$ there is an orbital $\Omega_{i}$ such that $l$-var $A\left(\Omega_{i}\right) \supseteq$ $\mathcal{A}^{n}$. In turn, this is equivalent to $g$-var $A\left(\Omega_{i}\right) \supseteq \mathfrak{S}_{n}$; and the corollary follows because $\mathfrak{G}$ is the join of all $\mathfrak{S}_{n}$ (any free group is a subdirect product of solvable groups).

THEOREM 2.9. $l$-var $A(\Omega)$ is either $\mathcal{L}, \mathcal{N}$, or $\mathfrak{A}^{n}$ for some $n$.

PROOF. Since the named varieties form a complete tower, and since $A(\Omega)$ belongs to a variety $\mathcal{V}$ iff the restriction of $A(\Omega)$ to each of its orbitals belongs to $\mathcal{V}$, it suffices to prove the theorem assuming $A(\Omega)$ has just one orbital. If some primitive component is not regular, then $l$-var $A(\Omega)=\mathcal{L}$ [5]. If all primitive components are regular then $A(\Omega) \in \mathcal{N}(\operatorname{Read}[\mathbf{1 1}])$. The theorem now follows from Corollary 2.6 and the fact that $\mathcal{N}$ is the join of all $A^{n}$.

THEOREM 2.10. $g$-var $A(\Omega)$ is either $\mathfrak{G}$ or $\mathfrak{S}_{n}$ for some $n$.

PROOF. Immediate from Theorem 2.9 and Corollaries 2.7 and 2.8.

COROllary 2.11. $A(\Omega) \in \mathscr{A}^{n}$ iff $A(\Omega) \in \mathfrak{S}_{n}$.

It follows from Theorem 2.5 that if $l$-var $A(\Omega)=\mathcal{N}$ then, for each $n, A(\Omega)$ contains an $l$-subgroup isomorphic to $\mathrm{Wr}^{n} Z$. In $\S 5$ we need the following strong version of this fact.

LEMMA 2.12. If $l$ - $\operatorname{var} A(\Omega)=\mathcal{N}$ then $A(\Omega)$ contains l-subgroups $G_{n}, n=$ $1,2, \ldots$, such that $G_{n} \approx \mathrm{Wr}^{n} Z$ and $\operatorname{supp} G_{i} \cap \operatorname{supp} G_{j}=\emptyset$ if $i \neq j$.

Proof. Case 1. Suppose, for every orbital $\Omega_{\lambda}, l$-var $A\left(\Omega_{\lambda}\right) \neq \mathcal{N}$. Inductively, suppose $\mathrm{Wr}^{i} Z \approx G_{i} \subseteq A\left(\Omega_{\lambda_{i}}\right), i=1,2, \ldots, n$, with $\Omega_{\lambda_{i}} \neq \Omega_{\lambda_{j}}$ when $i \neq j$. Then $l$-var $A\left(\Omega_{\lambda_{i}}\right)=A^{m_{i}}$, so there exists $\Omega_{\lambda_{n+1}}$ such that $l$-var $A\left(\Omega_{\lambda_{n+1}}\right) \nsupseteq A^{m_{i}}, i=$ $1,2, \ldots, n$. Hence $\Omega_{\lambda_{n+1}}$ is different from all $\Omega_{\lambda_{i}}, i \leq n$, and $A\left(\Omega_{n+1}\right)$ contains an $l$-subgroup $G_{n+1}$ isomorphic to $\mathrm{Wr}^{n+1} Z$ by Theorem 2.5 .

Case 2. Suppose $l$-var $A\left(\Omega_{\lambda}\right)=\mathcal{N}$ for some orbital $\Omega_{\lambda}$. 
Subcase 2a. Suppose $\Omega_{\lambda}$ has no largest proper natural congruence. Inductively, suppose $\mathrm{Wr}^{i} Z \approx G_{i} \subseteq A\left(\Omega_{\lambda}\right), i=1,2 \ldots, n$, with each $G_{i}$ having a join-irreducible generator $g_{i}$ for its uppermost copy of $Z$. Then $\operatorname{supp} g_{i}$ is a congruence class $B_{i}$ (because $A\left(\Omega_{i}\right) \in \mathcal{N}$; see $\left.[\mathbf{1 1}]\right)$. There exists a join irreducible $g_{n+1}^{\prime}$ which moves $B_{n}$. Let $\operatorname{supp} g_{n+1}^{\prime}=B_{n+1}^{\prime}$. Then the conjugates of $G_{n}$ by powers of $g_{n+1}^{\prime}$ have disjoint support, so $A\left(\Omega_{\lambda}\right)$ contains their full direct product which, together with $g_{n+1}^{\prime}$, generates an $l$-subgroup $G_{n+1}^{\prime}$ isomorphic to $\mathrm{Wr}^{n+1} Z$. There is a proper congruence class $B \supseteq B_{n+1}^{\prime}$. Let $g$ be any element of $A\left(\Omega_{\lambda}\right)$ which moves $B$. Let $G_{n+1}=g^{-1} G_{n+1}^{\prime} g$.

Subcase $2 \mathrm{~b}$. Suppose $\Omega_{\lambda}$ has a largest proper natural congruence $C$. Then $A\left(\Omega_{\lambda}\right)$ induces $A(C)$ on each $C$ class $C$. It cannot be that $l$-var $A(C) \subseteq A^{n}$ for a fixed $n$ and all $C$, for then $A\left(\Omega_{\lambda}\right) \in A^{n+1}$. Hence for each $n$ there exists a $C$ class $C_{n}$ such that $\mathrm{Wr}^{n} Z \subseteq A\left(C_{n}\right)$, and we may assume $C_{n} \neq C_{m}$ when $n \neq m$, since each $C$ class has infinitely many isomorphic translates. The proof can now be completed just as in Case 1.

3. The structure of transitive $A(\Omega) \in A^{n}$. Ohkuma [10] showed that if $A(\Omega)$ is uniquely transitive (for each $\alpha, \beta \in \Omega$ there exists a unique $g \in A(\Omega)$ such that $\alpha g=\beta)$, then $A(\Omega)$ is isomorphic to a subgroup of the additive ordered group of real numbers and $\Omega \approx A(\Omega)$ as ordered sets. Nonzero subgroups of the reals with this property have since been called Ohkuma groups and extensively studied ([3]; see also Glass [2]). Since it is easily seen that a transitive abelian $A(\Omega)$ must be uniquely transitive, Ohkuma's result may be stated as follows: if $A(\Omega)$ is transitive and nontrivial, then $l$-var $A(\Omega)=\AA$ iff $\Omega$ is an Ohkuma group, and in this case $A(\Omega) \approx \Omega$. In this section we study the analogous problem when $l$-var $A(\Omega)=A^{n}$.

The heart of the main theorem of this section is contained in the following technical lemma.

LEMMA 3.1. Let $O_{1}, O_{2}, \ldots, O_{n}$ be Ohkuma groups, and $\Omega=O_{1} \times O_{2} \times \cdots \times O_{n}$ ordered lexicographically from the right. Then (i) $\Omega$ is not isomorphic to any proper segment of itself, and (ii) no proper initial segment of $\Omega$ is isomorphic to any proper final segment of $\Omega$.

PROOF. We deal first with the case $n=1$. The result is clearly true if $\Omega=$ $O_{1} \approx Z$. Since all other (noncyclic) subgroups of the reals are dense, we assume $\Omega=O_{1}$ is a dense subgroup of the reals. If $\phi: \Omega \rightarrow \Omega^{\prime}$ is an isomorphism (of ordered sets) where $\Omega^{\prime}$ is a proper segment of $\Omega$, let $f$ be any nontrivial member of $A(\Omega)$. Then $\phi^{-1} f \phi$ is a nontrivial member of $A\left(\Omega^{\prime}\right)$, which can be extended to $f^{\prime} \in A(\Omega)$, fixing all points of $\Omega \backslash \Omega^{\prime}$. This contradicts the unique transitivity of $A(\Omega)$, hence (i) holds.

Since $\Omega$ is a dense subset of the real numbers, each initial segment of $\Omega$ has the form $(-\infty, \alpha)=\{x \in \Omega: x<\alpha\}$, where $\alpha$ is a real number (not necessarily in $\Omega$ ). Likewise, each final segment has the form $(\beta, \infty)$. Suppose $\psi:(-\infty, \alpha) \approx(\beta, \infty)$. Let $g$ be any negative member of $A(\Omega)$. Then $(-\infty, \alpha) \approx(-\infty, \alpha g)$, and if we let $\beta^{\prime}=\alpha g \psi$, there is an isomorphism $\phi:\left(\beta, \beta^{\prime}\right) \approx(\beta, \infty)$, which can be extended to an isomorphism of the real intervals $\left(\beta, \beta^{\prime}\right) \approx(\beta, \infty)$. Because $\Omega$ is dense, there exists a positive $t \in A(\Omega)$ such that $\beta<\beta t<\beta^{\prime}$. We may also consider $t$ extended to a map from the reals to the reals. Because both $t$ and $\phi$ preserve order, their extensions are continuous. Since $\beta t<\beta^{\prime}=\beta \phi$ and $\beta^{\prime} t<\infty=\beta^{\prime} \phi$, there exists a 
real number $\gamma$ such that $\gamma t=\gamma \phi$ and $\beta<\gamma<\beta^{\prime}$. Now define $f: \Omega \rightarrow \Omega$ by

$$
x f= \begin{cases}x & \text { if } x<\beta, \\ x \phi & \text { if } \beta \leq x \leq \beta^{\prime}, \\ x t & \text { if } \beta^{\prime}<x .\end{cases}
$$

Then $f \in A(\Omega)$. But $f$ fixes some points without fixing all, denying unique transitivity of $A(\Omega)$. Hence (ii) is satisifed. This takes care of the case $n=1$.

Now let $\Omega=O_{1} \times O_{2} \times \cdots \times O_{n}, n>1$, and suppose the result is true for $\Lambda=O_{1} \times O_{2} \times \cdots \times O_{n-1}$. Let $C_{n}$ be the equivalence relation on $\Omega$ given by $\left(a_{1}, a_{2}, \ldots, a_{n}\right) C_{1}\left(b_{1}, b_{2}, \ldots, b_{n}\right)$ iff $a_{n}=b_{n}$. If $\phi: \Omega \rightarrow \Omega^{\prime}$ is an isomorphism of $\Omega$ onto a proper segment of itself and $\phi$ preserves $C_{n}$ classes, then $\phi$ induces an isomorphism of $O_{n}$ onto a proper segment of itself, contradicting the result for $n=1$. Hence for some $C_{n}$ class $x C_{n}, x \phi C_{1} \neq x C_{n} \phi$. Each of these two classes is a convex segment of $\Omega$ and is isomorphic to $\Lambda$. Further, $x \phi$ belongs to both. Hence either one is contained in the other, or they overlap so that an initial segment of one is a final segment of the other. But each of these possibilities contradicts the inductive assumption about $\Lambda$. Hence (i) is satisfied by $\Omega$. A similar argument shows that (ii) is satisfied, completing the proof of the lemma.

THEOREM 3.2. If $A(\Omega)$ is transitive,

$$
l \text {-var } A(\Omega)=A^{n} \quad \text { iff } \Omega \approx O_{1} \times O_{2} \times \cdots \times O_{n},
$$

a lexicographically ordered product of Ohkuma groups; in this case $A(\Omega) \approx O_{1} \operatorname{Wr} O_{2}$ $\mathrm{Wr} \cdots \mathrm{Wr} O_{n}$.

PROOF. If $l$-var $A(\Omega)=A^{n}$, then there are exactly $n$ primitive components $O_{i}$ and are all regular by Corollary 2.6. But a transitive regular primitive component must be an Ohkuma group (see Glass [2]). Hence $A(\Omega)$ can be embedded in the wreath product $O_{1} \mathrm{Wr} O_{2} \mathrm{Wr} \cdots \mathrm{Wr} O_{n}$, which acts as an ordered permutation group on the set $O_{1} \times O_{2} \times \cdots \times O_{n}$ ordered lexicographically from the right. In the embedding $\Omega \approx O_{1} \times O_{2} \times \cdots \times O_{n}$, however, so $A(\Omega) \approx O_{1} \mathrm{Wr} O_{2} \mathrm{Wr} \cdots \mathrm{Wr} O_{n}$.

For the converse, suppose that $O_{1}, O_{2}, \ldots, O_{n}$ are given Ohkuma groups and $\Omega=O_{1} \times O_{2} \times \cdots \times O_{n}$. We claim that each of the relations $C_{i}$, given by $\left(a_{1}, a_{2}, \ldots, a_{n}\right) C_{i}\left(b_{1}, b_{2}, \ldots, b_{n}\right)$ iff $a_{j}=b_{j}$ for all $j \geq i$, is a congruence for $A(\Omega)$. This is the case because each of the $C_{i}$ classes is isomorphic to the set $\Omega=O_{1} \times O_{2} \times \cdots \times O_{i-1}$, to which Lemma 3.1 applies. It follows that the primitive components of $A(\Omega)$ are $A\left(O_{i}\right) \approx O_{i}$, each of which is nontrivial and regular, so $l$-var $A(\Omega)=A^{n}$ by Corollary 2.6.

The case of transitive $A(\Omega)$ with $l$-var $A(\Omega)=\mathcal{N}$ appears to be much more complicated. It is still true, just as before, that $\Omega$ is embedded in a lexicographically ordered product of Ohkuma groups, but the embedding need not be onto. Worse yet, even the full product of Ohkuma groups need not have the natural congruences. For example, $\cdots \times Z \times Z \times Z$ is isomorphic to the set of irrational real numbers, which has no proper natural congruence.

4. The group structure of arbitrary $A(\Omega) \in A^{n}$. A theorem of Chang and Ehrenfeucht $[\mathbf{1}]$ states that $A(\Omega) \in A$ iff $A(\Omega)$, as a group, is a full direct product of subgroups of the real numbers $R$. In order to extend this to higher powers, we define a group $G$ to be of wreal height $\leq n$ inductively as follows. If $n=0, G=\{e\}$; 
in general, $G \approx\left\{G_{\lambda}\right\} \mathrm{Wr}(H, \Lambda)$, where $H$ is a subgroup of $R$ acting regularly on $\Lambda$, a union of orbits of $H$ in $R, G_{\lambda}=\prod_{k} G_{\lambda, k}$, and each $G_{\lambda, k}$ is a group of wreal height $\leq n-1$. Note that $G$ has wreal height $\leq 1$ iff $G$ is isomorphic to a subgroup of $R$. We will show that $A(\Omega) \in A^{n}$ iff $A(\Omega)$ if a full direct product of groups of wreal height $\leq n$.

THEOREM 4.1. If $A(\Omega) \in A^{n}$, then $A(\Omega)$ is a full direct product of groups of wreal heighi $\leq n$.

PROOF. It suffices to prove that if $A(\Omega)$ has just one orbital and belongs to $A^{n}$, then $A(\Omega)$ is a group of wreal height $\leq n$. The theorem is trivially true if $n=0$. In the general case, by Corollary 2.6 and Theorem 2.9, $A(\Omega)$ has exactly $m \leq n$ nontrivial primitive components, and each is regular. If $H$ is the component corresponding to the uppermost covering pair of natural congruences $\left(C_{i}, C^{i}\right)$, then $H$ is a subgroup of $R$ acting on $\Lambda$, a union of its orbits. On each of the $C_{i}$ classes $\theta_{\lambda}, A(\Omega)$ induces $A\left(\theta_{\lambda}\right) \in \mathscr{A}^{n-1}$, and so by induction $A\left(\theta_{\lambda}\right)$ is a product of groups of wreal height $\leq n-1$. Since $A(\Omega) \approx\left\{A\left(\theta_{\lambda}\right)\right\} \mathrm{Wr}(H, \Lambda), A(\Omega)$ has wreal height $\leq n$.

THEOREM 4.2. If $G$ is a product of groups of wreal height $\leq n$, then, for some $\Omega, G \approx A(\Omega)$ and $A(\Omega) \in \mathcal{A}^{n}$.

ProOF. The theorem is trivial for $n=0$. We proceed by induction. We may assume $G$ has wreal height $\leq n$, for if $G=\prod P_{i}$ and each $P_{i}$ has wreal height $\leq n$, and if we know $P_{i} \approx A\left(\Omega_{i}\right) \in \mathcal{A}^{n}$, let $\left\{\pi_{i}\right\}$ be ordinals each greater than $\bigvee_{i}\left|P_{i}\right|$, and all different, and let $\Omega_{i}^{\prime}=\pi_{i} \times \Omega_{i}$ ordered lexicographically from the right. Let $\Omega$ be the disjoint union of all $\Omega_{i}^{\prime}$ ordered in any way so that each $\Omega_{i}^{\prime}$ (as previously ordered) is convex. Then $A(\Omega)=\prod A\left(\Omega_{i}^{\prime}\right) \approx \prod A\left(\Omega_{i}\right)=G$. Hence, we now assume $G$ has wreal height $\leq n$ and is a wreath product as in the definition. By induction, each $G_{\lambda} \approx A\left(\theta_{\lambda}\right) \in A^{n-1}$. Let $\Omega^{*}=\left\{(\sigma, \lambda) \in\left(\bigcup \theta_{\lambda}\right) \times \Lambda: \sigma \in \theta_{\lambda}\right\}$. Then

$$
G=\left\{g \in A\left(\Omega^{*}\right):(\sigma, \lambda) g=\left(\sigma g_{\lambda}, \bar{g}\right), g_{\lambda} \in A\left(\theta_{\lambda}\right), \bar{g} \in H\right\} .
$$

Suppose, in the first case, that $H$ is a proper subgroup of $R$. We consider the regular action $(H, R)$. Following Chang and Ehrenfeucht, for each orbit (coset) $J_{i}$ of $H$ in $R$, we let $\pi_{i}$ be an ordinal greater than $\bigvee_{\lambda}\left|\theta_{\lambda}\right|$ and all $\pi_{i}$ different. If we replace each real number $r$ by the ordinal $\pi_{i}$ when $r \in J_{i}$, we get a totally ordered set $T$ and a natural action $(H, T)$ such that $H=A(T)$ (see [1]). Moreover, we can assume $\Lambda \subseteq R \subseteq T$. For $\lambda \in \Lambda$ we already have $\theta_{\lambda}$ defined. For the other $t \in T \backslash \Lambda$, let $\theta_{t}$ be a single point, so $A\left(\theta_{t}\right)=\{e\}$. Then

$$
G \approx\left\{A\left(\theta_{t}\right): t \in T\right\} \mathrm{Wr}(A(T), T) .
$$

Let $\Lambda=\left\{(\sigma, t) \in\left(\bigcup \theta_{t}\right) \times T: \sigma \in \theta_{t}\right\}$ be the set acted on by this wreath product. Clearly each $\theta_{t} \times\{t\}$ is a congruence class for $A(\Omega)$ because if $x<y$ with $x, y \in$ $\theta_{t} \times\{t\}$, then $|(x, y)|<\pi_{i}$ for all $i$, while if $x \in \theta_{t} \times\{t\}$ and $y \in \theta_{t^{\prime}} \times\left\{t^{\prime}\right\}$ with $t \neq t^{\prime}$, then some ordinal $\pi_{i}$ lies between $x$ and $y$. It follows that

$$
A(\Omega) \approx\left\{A\left(\theta_{\lambda}\right)\right\} \mathrm{Wr}(A(T), T) \approx G .
$$

In the second case, $H=R$. Then $H$ is isomorphic to a proper subgroup $H^{\prime}$ of $R$, and as permutation groups (thought not as ordered permutation groups), $(H, H) \approx\left(H^{\prime}, H^{\prime}\right)$. It follows that $G \approx\left\{G_{h}\right\} \mathrm{Wr}(H, H) \approx\left\{G_{h^{\prime}}\right\} \mathrm{Wr}\left(H^{\prime}, H^{\prime}\right)$, and we proceed as in the first case. 
Finally, observe that it follows from induction that any group of wreal height $\leq n$ belongs to the solvable variety $\mathfrak{S}_{n}$. Hence, by Corollary 2.11 , if $A(\Omega)$ is a product of groups of wreal height $\leq n$, then $A(\Omega) \in A^{n}$.

COROLLARY 4.3. If $G$ is a group, there exists $\Omega$ such that $G \approx A(\Omega) \in A^{n}$ iff $G$ is a full direct product of groups of wreal height $\leq n$.

COROllary 4.4 (CHANG AND Ehrenfeucht $[\mathbf{1}]$ ). If $G$ is a group, there exists $\Omega$ such that $G \approx A(\Omega) \in A$ iff $G$ is a full direct product of subgroups of the real numbers.

5. Free subgroups of $A(\Omega)$. The following lemma is only a slight modification of a theorem of J. Mycielski applied to the special case of $l$-groups. We invoke Mycielski's theorem in the proof.

LEMMA 5.1. Let $\mathcal{V}$ be a variety of groups (or of l-groups). Suppose there are subgroups (or l-subgroups) $G_{i} \subseteq A(\Omega), i=1,2, \ldots$, such that $\operatorname{supp} G_{i} \cap \operatorname{supp} G_{j}=\emptyset$ if $i \neq j$, and whenever $(w=e)$ is not a law of $\mathcal{V}$ (where $w$ is a word in the free group (or free l-group) on $\left.x_{1}, x_{2}, \ldots\right)$, then there exists $i=i(w)$ such that if $j \geq i, G_{j}$ does not satisfy $(w=e)$. Then $A(\Omega)$ contains $\prod G_{i}$ which contains a free $\mathcal{V}$ group (or l-group) on $2^{\aleph_{0}}$ generators.

Proof. Clearly $A(\Omega) \supseteq \prod G_{i}$. We first show $\prod G_{i}$ has a free subgroup $(l$ subgroup) on $\aleph_{0}$ generators. List all those words $w_{1}, w_{2}, \ldots$ such that $\left(w_{i}=e\right)$ is not a law of $\mathcal{V}$. Inductively, there exists $i(n)>i(n-1)$ such that $G_{i(n)}$ does not satisfy $\left(w_{n}=e\right)$; that is, there are $g(n, 1), g(n, 2), \ldots \in G_{i(n)}$ such that $w_{n}(\bar{g}(n,-)) \neq e$. Let $g_{i}=(g(1, i), g(2, i), \ldots) \in \prod G_{n}$. Then $\left\{g_{1}, g_{2}, \ldots\right\}$ is free.

Next, partition $Z^{+}=P_{1} \dot{\cup} P_{2} \cup \ldots$ into an infinite number of infinite sets. Then for each $j,\left\{G_{i}: i \in P_{j}\right\}$ satisfies the hypotheses of the lemma. Hence $G_{j}^{\prime}=$ $\prod_{i \in P_{j}} G_{i}$ contains a free $\mathcal{V}$ subgroup (or $l$-subgroup) on a countable set. Moreover, $\operatorname{supp} G_{j}^{\prime} \cap \operatorname{supp} G_{k}^{\prime}=\emptyset$ when $j \neq k$, so $A(\Omega) \supseteq \prod G_{j}^{\prime}$. Mycielski's theorem ([8, Corollary 3] and the ensuing discussion) implies that $\prod G_{j}^{\prime}$ contains a free $\mathcal{V}$ subgroup (or $l$-subgroup) on $2^{\aleph_{0}}$ generators.

THEOREM 5.2. If $l$-var $A(\Omega)=A^{n}$ then $A(\Omega)$ contains a free $A^{n-1}$ l-subgroup on $2^{\aleph_{0}}$ generators.

ProOF. We may suppose $A(\Omega)$ has just one orbital and a maximal proper natural congruence $C$ (Corollary 2.6). For some $C$ class $C, l$-var $A(C)=A^{n-1}$ (otherwise $A(\Omega) \in A^{n-1}$ ). There are infinitely many disjoint translates of $C$, so $A(\Omega)$ contains $l$-subgroups $G_{i} \approx \mathrm{Wr}^{n-1} Z$ satisfying the hypotheses of Leinma 5.1 for $\mathcal{V}=\AA^{n-1}$ (by Lemma 2.3).

THEOREM 5.3. If $g$ - $\operatorname{var} A(\Omega)=\mathfrak{S}_{n}$, then $A(\Omega)$ contains a free $\mathfrak{S}_{n-1}$ subgroup on $2^{\aleph_{0}}$ generators.

Proof. The hypothesis is equivalent to that of Theorem 5.2 (by Corollary 2.6). In the proof of Theorem 5.2, since $l$-var $A(C)=A^{n-1}, g$-var $A(C)=\mathfrak{S}_{n-1}$ by Corollary 2.6, and the hypotheses of Lemma 5.1 are satisfied for $\mathcal{V}=\mathfrak{S}_{n-1}$.

THEOREM 5.4. If $l$ - $\operatorname{var} A(\Omega)=\mathcal{N}$ then $A(\Omega)$ contains a free $\mathcal{N} l$-subgroup on $2^{\aleph_{0}}$ generators. 
Proof. $\mathcal{N}$ is generated by the set of all $\mathrm{Wr}^{n} Z$ since $\mathcal{N}$ is the join of all $A^{n}$ (and using Lemma 2.3). From Lemma 2.12 the hypotheses of Lemma 5.1 are satisfied with $\mathcal{V}=\mathcal{N}$.

THEOREM 5.5. If $l$ - $\operatorname{var} A(\Omega)=\mathcal{L}$ then $A(\Omega)$ contains a free $l$-subgroup on $2^{\aleph_{0}}$ generators.

Proof. Some primitive component of $A(\Omega)$ is not regular and so is 0 -2-transitive on one of its orbits (see remarks preceding Lemma 2.1). Let $\alpha<\beta$ be points of that orbit. Then the restriction of $A(\Omega)$ to the interval $(\alpha, \beta)$ satisfies no nontrivial l-group law [5]. Hence, choosing disjoint intervals, we have $l$-subgroups $G_{i} \subseteq A(\Omega)$ satisfying the hypotheses of Lemma 5.1 for $\mathcal{V}=\mathcal{L}$.

THEOREM 5.6. If $g$ - $\operatorname{var} A(\Omega)=\mathfrak{G}$, then $A(\Omega)$ contains a free subgroup on $2^{\aleph_{0}}$ generators.

Proof. We have either $l$-var $A(\Omega)=\mathcal{N}$ or $l$-var $A(\Omega)=\mathcal{L}$ by Corollary 2.8. In either case $A(\Omega)$ has subgroups $G_{n} \approx \mathrm{Wr}^{n} Z$ satisfying the hypotheses of Lemma 5.1 for $\mathcal{V}=\mathfrak{G}$ (by Lemmas 2.2, 2.3 and 2.12).

\section{REFERENCES}

1. C. C. Chang and A. Ehrenfeucht, A characterization of abelian groups of automorphisms of a simply ordering relation, Fund. Math. 51 (1962), 141-147.

2. A. M. W. Glass, Ordered permutation groups, London Math. Soc. Lecture Notes Ser., Vol. 55, Cambridge Univ. Press, London and New York, 1981.

3. A. M. W. Glass, Y. Gurevich, W. C. Holland and S. Shelah, Rigid homogeneous chains, Math. Proc. Cambridge Philos. Soc. 89 (1981), 7-17.

4. A. M. W. Glass, W. C. Holland and S. H. McCleary, The structure of $l$-group varieties, Algebra Universalis 10 (1980), 1-20.

5. W. C. Holland, The largest proper variety of lattice ordered groups, Proc. Amer. Math. Soc. $\mathbf{5 7}$ (1976), 25-28.

6. J. Martinez, Varieties of lattice-ordered groups, Math. Z. 137 (1974), 256-284.

7. S. H. McCleary, The structure of intransitive ordered permutation groups, Algebra Universalis 6 (1976), 229-255.

8. J. Mycielski, Almost every function is independent, Fund. Math. 81 (1973), 43-48.

9. H. Neumann, Varieties of groups, Ergeb. Math. Grenzgeb., No. 37, Springer-Verlag, 1967.

10. T. Ohkuma, Sur quelques ensembles ordonnés linéarment, Fund. Math. 43 (1955), 326-337.

11. J. A. Read, Wreath products of non-overlapping lattice-ordered groups, Canad. Math. Bull. 17 (1975), 713-722.

Department of Mathematics, Bowling Green State University, Bowling GREEN, OHIO 43403 